\title{
Dangerous implications of a minimum length in quantum gravity
}

\author{
Cosimo Bambi ${ }^{1,2}$ and Katherine Freese ${ }^{2}$ \\ ${ }^{1}$ Department of Physics and Astronomy, Wayne State University, Detroit, MI 48201, USA \\ ${ }^{2}$ Michigan Center for Theoretical Physics, University of Michigan, Ann Arbor, MI 48109, USA
}

Received 15 May 2008, in final form 4 July 2008

Published 16 September 2008

Online at stacks.iop.org/CQG/25/195013

\begin{abstract}
The existence of a minimum length and a generalization of the Heisenberg uncertainty principle seem to be two fundamental ingredients required in any consistent theory of quantum gravity. In this paper, we show that they would predict dangerous processes which are phenomenologically unacceptable. For example, long-lived virtual super-Planck mass black holes may lead to rapid proton decay. Possible solutions of this puzzle are briefly discussed.
\end{abstract}

PACS numbers: 03.65.Ta, 04.60.Bc

\section{Introduction}

The search for a theory of quantum gravity is a sort of holy grail in physics; yet, despite a great deal of effort, even the very meaning of quantum spacetime is still not clear. The difficulties are essentially related to the quite peculiar nature of the gravitational force. At present we can at most rely on a small set of model-independent features which are expected to hold in any consistent framework of quantum gravity. In particular, from quite general grounds, we can deduce the existence of a minimum length and a generalization of the Heisenberg uncertainty principle of quantum mechanics [1] (for a review, see [2]). The argument is basically the following. In quantum mechanics, the accuracy on the position of a particle is limited by the uncertainty on its momentum by the well known rule $\Delta x \gtrsim 1 / \Delta p$. On the other hand, in general relativity a certain amount of energy cannot be localized in a region smaller than the one defined by its gravitational radius, so $\Delta x \gtrsim L_{\mathrm{Pl}}^{2} \Delta p$, where $L_{\mathrm{Pl}}=G_{N}^{1 / 2} \sim 10^{-33} \mathrm{~cm}$ is the Planck length. Now, combining the two results, we conclude that there must exist a minimum observable length

$$
\Delta x \gtrsim \max \left(\frac{1}{\Delta p} ; L_{\mathrm{Pl}}^{2} \Delta p\right) \gtrsim L_{\mathrm{Pl}}
$$

Such a result can be summarized in the so-called generalized uncertainty principle, which can be deduced from more sophisticated gedanken experiments in different frameworks [1] and reads

$$
\Delta x \gtrsim \frac{1}{\Delta p}+\alpha L_{\mathrm{Pl}}^{2} \Delta p,
$$


where $\alpha$ is some positive and dimensionless model-dependent coefficient, usually assumed to be of order one. As one can easily suspect at this point, it is also possible to derive a generalized time-energy uncertainty relation $[2,3]$

$$
\Delta t \gtrsim \frac{1}{\Delta E}+\beta L_{\mathrm{Pl}}^{2} \Delta E
$$

where $\beta$ is a new positive model-dependent parameter.

\section{The paradox}

In quantum mechanics, the nature of the relation between time and energy $\Delta t \Delta E \gtrsim 1$ is quite different from the one between position and momentum $\Delta x \Delta p \gtrsim 1: t$ is a parameter and not an operator, the corresponding formula does not arise from 'first principles' (i.e. from the commutation relation) and in the end it is not always true, see, e.g., the discussion in [4]. Nevertheless, in most physical processes it works and is capable of providing a simple interpretation of several phenomena. For example, it provides a mechanism to evaluate the lifetime of an excited state from its energy width in atomic and nuclear physics. In quantum field theory, it allows for an interpretation according to which a virtual particle of energy $E$ can be produced out of vacuum for a time $1 / E$. Even if we still do not have any reliable theory of quantum gravity, we can expect that such general considerations are common to any quantum theory and are not ruled out by including the effects of gravitational interaction (or at least this is the only thing we can do at present!). Hence, if we take equation (3) seriously, we are induced to assert that in quantum gravity any excited state of energy $E$ can be produced out of vacuum for a time

$$
\tau \sim \max \left(\frac{1}{E} ; L_{\mathrm{Pl}}^{2} E\right) .
$$

The result is that super-Planckian excitations can exist for a very long time. This fact presents a paradox, because it implies that the contribution from heavier and heavier states of the theory should be more and more important, even in low-energy physical processes. Such a situation destroys the success of our current framework of particle physics and predicts even more dangerous events, which are clearly inconsistent with the universe in which we live.

One might worry about the contribution of long-lived virtual (1) classical macroscopic objects, (2) sub-Planck mass particles with super-Planckian energies or (3) super-Planck mass particles to dangerous physical processes; we will show that it is the third category that is really problematic.

In principle, equation (3) could permit classical macroscopic virtual objects to live for very long times, because their mass is much larger than the Planck mass $M_{\mathrm{Pl}} \sim 10^{-5} \mathrm{~g}$. However, such a possibility is not dangerous, because classical objects are extremely tidy states, so they should be suppressed by a huge entropy factor. This suppression is familiar in a different context: in particle physics accelerators, it is extremely unlikely that large classical objects such as magnetic monopoles or sphalerons could be produced, though a rigorous calculation has not been performed ${ }^{3}$. For this reason we can neglect the contribution of virtual

3 For example, monopole-antimonopole annihilation into all possible particles should be quite efficient, with a cross section $\sigma \sim\left(m_{M}\right)^{-2}$ and a reaction probability $\Gamma \sim m_{M}$, where $m_{M}$ is the monopole mass and the only energy scale in the problem. One might expect the inverse process, the assembly of multiple particles into a monopole-antimonopole pair to proceed with the same probability. However, the number of particles produced by monopole-antimonopole annihilation is expected to be of order $1 / \alpha \sim 100$ and in a very coherent state; such a coherent state is not likely ever to be created in a particle collision, so that the production of monopoles in accelerators is very unlikely ever to take place. 
classical objects: the probability to produce them is not determined mainly by the uncertainty principle, but by a huge entropy factor which makes their creation hard even when the process is classically allowed. For more details on the point, see, e.g., [5] and references therein. One could also argue that the crucial ingredient of the generalization of the Heisenberg uncertainty principle is the energy density, rather than the total mass [6], and the one of macroscopic objects is typically much smaller than the 'critical value' which is necessary for the formation of a horizon.

Second, one might worry about the contribution of virtual sub-Planck mass particles with energy beyond the Planck scale to dangerous physical processes. Here quantum field theory surely breaks down, computations become unreliable, and it would be difficult to predict the implications. However, as is usually done, we could solve the problem by asserting that the Planck scale represents a cut-off for the energy of an elementary particle. For example, in some frameworks the Planck energy and/or the Planck momentum cannot be reached by elementary objects [7]. If this does not occur, the modified measure in momentum space can do the job, acting as a soft cut-off: basically, equations (2) and (3) can be seen as a redefinition of the Planck constant $h$, which sets the infinitesimal volume of the phase space. This affects loop integration with a correction of the form ${ }^{4}$

$$
\int \frac{\mathrm{d}^{4} p}{(2 \pi)^{4}} \rightarrow \int \frac{\mathrm{d}^{4} p}{(2 \pi)^{4}\left(1+\alpha / L_{\mathrm{Pl}}^{2} p^{2}\right)^{4}}
$$

and suppresses high-energy contributions.

The true paradox is instead related to the contribution of super-Planck mass virtual black holes: since in any quantum theory one expects to find the fundamental excitations of the theory, virtual black holes are supposed to be present in the mass spectrum of the (yet unknown) theory of quantum gravity. The standard argument for them is the spacetime foam picture $[8,9]$. Using a semiclassical approach, one considers the path integral for $N$ non-interacting black holes and finds

$$
Z \sim \int_{0}^{\infty} \mathrm{d} M \sum_{N=0}^{\infty} \frac{1}{N !}\left(\frac{V}{L_{\mathrm{Pl}}^{3}}\right)^{N} \exp \left(-4 \pi L_{\mathrm{Pl}}^{2} N M^{2}\right)
$$

where $V$ is a normalization volume. Let us note that the integration is over all the black hole masses, that is from zero to infinity. Nevertheless, the contribution of heavier and heavier black holes is more and more suppressed and since $L_{\mathrm{Pl}}$ acts as natural cut-off, one eventually finds that the spacetime is essentially filled with Planck mass black holes which live for about one Planck time.

Assuming the validity of the generalized uncertainty principle, the picture changes dramatically. Indeed, in any quantum theory at zero temperature, the contribution of virtual states is suppressed only by their limited existence, which is controlled by the time-energy uncertainty relation and goes to zero in the classical limit. Since the generalized uncertainty principle says that, beyond the Planck energy, the violation of a larger and larger amount of energy is more and more unsuppressed, the contribution of super-Planck mass virtual black holes is presumably more important than the contribution of Planck size ones. Heavy (i.e. with a mass $M_{\mathrm{BH}} \gg M_{\mathrm{Pl}}$ ) Schwarzschild black holes, and more generally heavy non-extremal black holes, might still be considered as semiclassical objects, exponentially suppressed by a huge factor (the point is however controversial, see, e.g., [10]). It is instead common belief that extremal black holes can be thought of as ordinary particles [11]. For example, extremal

4 The exact expression is however model dependent: it is determined by the canonical commutation relation $[x, p]$ which are not unique, i.e. there are infinite choices leading to equation (2). 
Reissner-Nordström black holes have vanishing Hawking temperature, are expected to be stable, and can contribute here. The modified measure in momentum space cannot solve the problem, just because it is not the ingredient which determines the density of black hole states and it is not clear how a minimum length scale can act as cut-off in this case. So, if we take literally equation (3), it seems very hard to prevent pair production of virtual extremal Reissner-Nordström black holes. Then, since black holes can violate global quantum numbers such as baryon and lepton number [12], ordinary matter should be unstable and quickly decay.

\section{Proton decay}

An unstable particle of mass $m$ usually decays into lighter particles with a lifetime $\sim\left(g^{2} m\right)^{-1}$, where $g$ is the coupling constant of the interaction responsible for the decay. This is for example the case of the $t$-quark, which decays into real $b$-quark and $W$ boson with a lifetime $\left(\alpha m_{t}\right)^{-1}$. On the other hand, if the process is kinematically forbidden, it proceeds through the emission of virtual particles and is therefore suppressed, making the particle lifetime increase. More precisely, if the process requires an intermediate heavy particle of mass $M$, it needs to violate energy conservation for a time $t \sim 1 / M$ in a volume $V \sim 1 / M^{n}$, where $n$ is the number of space dimensions. Using an effective quantum field theory, the process is described by a non-renormalizable operator suppressed by some power of $1 / M$, depending on the number of dimensions of the spacetime and on the kind of particles involved. This is quite a general rule which is independent of the interaction involved.

When gravity is taken into account, Planck mass virtual black holes are expected to induce proton decay (see, e.g., $[13,14]$ and references therein). In an effective field theory in $3+1$ dimensions, the process is described in the standard picture by a dimension 6 operator, since two quarks of the proton are converted into a quark (or an antiquark) and a charged lepton,

$$
\mathcal{O}_{6} \sim \frac{\psi \psi \psi \psi \psi}{M_{\mathrm{Pl}}^{2}} .
$$

The estimated proton lifetime in the standard framework (that is with the standard uncertainty principle) is

$$
\tau_{p} \sim \frac{M_{\mathrm{Pl}}^{4}}{m_{p}^{5}} \sim 10^{45} \mathrm{yr}
$$

and is consistent with present experimental limits, where several channels are bounded by $\tau_{p} \gtrsim 10^{33} \mathrm{yr}$.

Of course, the prediction in equation (8) must be revised in the case of the generalized uncertainty relations. Now, beyond the Planck scale, position and time uncertainties increase as the energy increases. The old suppression factors, proportional to some power of the inverse of the energy scale of the reaction (in the case of Planck mass black holes proportional to some power of $1 / M_{\mathrm{Pl}}$ ), should be replaced by the same power of the black hole gravitational radius $M_{\mathrm{BH}} / M_{\mathrm{Pl}}^{2}$, where $M_{\mathrm{BH}}$ is the black hole mass (which is now larger than $M_{\mathrm{Pl}}$ ). We can therefore guess that the proton lifetime should be

$$
\tau_{p} \sim \frac{M_{\mathrm{Pl}}^{8}}{m_{p}^{5} M_{\mathrm{BH}}^{4}} .
$$

This means that excited states heavier than roughly $10^{3} M_{\mathrm{Pl}}$ would induce proton decay which is far too rapid to be consistent with experiments.

Of course, the validity of an effective field theory to describe objects heavier than the Planck mass may be questionable. However we note that the semiclassical approach suggests 
that virtual black holes of every mass are produced in the spacetime foam and can thus give a nonzero contribution to the amplitude of physical processes. On the other hand, from the generalized uncertainty principle we can argue that the contribution of super-Planck mass virtual black holes have to be more important than the one of Planck mass black holes, because of the longer lifetime. Even if we do not have a rigorous framework, semiclassical results are usually believed to be at least capable of providing the basic features. Surely the description breaks down for $M_{\mathrm{BH}} \rightarrow+\infty$, but we will show in the following section that the rapid proton decay is mediated by virtual black holes for which the effective theory is likely still reliable. Lastly, we stress that the apparent paradox is a peculiar implication of the unsuppressed contributions of very heavy states. The problems of infinite pair production which are present in attempts to incorporate black hole remnants in an effective theory [15] are very different: in that case, the origin of the problems is due to the concept of remnant, because remnants can be a viable solution to the black hole information paradox only if there is an infinite amount of states below some finite mass (see, however, [16] for a different point of view). Here we do not need the existence of remnants and, without the generalized uncertainty principle, our description of virtual black holes would be phenomenologically acceptable.

\section{Possible solutions}

Even if equation (3) is deduced from heuristic considerations, the arguments supporting the picture sound quite robust and the new position-momentum and time-energy uncertainty relations look at least reasonable. In this case, it is not easy to suppress the contribution of virtual black holes: the simple replacement of the Heisenberg uncertainty principle with the generalized uncertainty principle leads to a paradox and we should figure out what is wrong. Before proceeding, we would like to stress that these black holes are always 'virtual', that is cannot be seen directly, since we cannot produce the super-Planckian energies required to create them. Nevertheless, they should be observed indirectly, through their contribution in low-energy physical events.

Broadly speaking, an elegant mechanism which is capable of forbidding (if exact) or reducing considerably (if approximate) dangerous processes otherwise inevitable is the introduction of a new symmetry. However, this is not so easy here: a global symmetry is expected to be violated by black holes, while an unbroken gauge symmetry demands a new massless gauge boson, which is questionable at best or more likely phenomenologically forbidden. Broken gauge symmetries also cannot work, because a charge related to the gauge boson of mass $m$ disappears without any trace inside the black hole, with a characteristic time $1 / m$ [17]; i.e., the quantum number associated with the symmetry is violated. At present, the only known possibility is to introduce a gauge symmetry which is broken to a discrete remnant [18]: in this case, even if the gauge boson acquires a mass, Gauss' law implies that when a charge related to the symmetry falls into the black hole, a surface integral which is an intrinsic feature of the asymptotic black hole state still survives and the quantum number is unchanged. An explicit realization can be found in [19], while for more general considerations see [20].

Another possibility could be to appeal to the peculiar (and not well understood) nature of non-extremal and extremal black holes: the former might be exponentially suppressed because they are semiclassical objects, the latter might be unable to violate global quantum numbers. For instance, although virtual long-lived extremal Reissner-Nordström black holes can come into existence, one might argue (based on the fact that they have zero temperature) that these black holes do not violate baryon number [12]. However, such an explanation cannot work for other extremal states: examples include the extremal black holes of the charged dilaton family, which can have nonzero temperature even if zero entropy. On the other hand, these 
extremal states may not exist at all, because we do not know if dilaton-like fields really exist in nature.

However, all these proposed solutions can at best avoid too rapid proton decay and other dangerous baryon or lepton violating processes. There is still the danger that heavy extremal black holes can participate in ordinary reactions in the standard model of particle physics that are thus unsuppressed. Then the branching ratios of ordinary processes would be altered, leading to disagreement with experiment. All the observed branching ratios are essentially in agreement with theoretical predictions, while black holes should change them, because the effective field theory we have outlined in this paper breaks down for very heavy black holes. To be more quantitative, we can consider a general process which is allowed in the standard model, for instance $e+v_{\mu} \rightarrow \mu+v_{e}$. Such a process should be possible with an intermediate virtual black hole as well and in that case it would be described by a dimension 6 operator like the one in equation (7). In the standard theory, $M$ is the Planck mass and the differential cross section is

$$
\frac{\mathrm{d} \sigma}{\mathrm{d} \Omega} \sim \frac{E^{2}}{M_{\mathrm{Pl}}^{4}} \sim|f(\theta)|^{2},
$$

where $f(\theta)$ is the scattering amplitude. On the other hand, in the case of the generalized uncertainty principle $M=M_{\mathrm{Pl}}^{2} / M_{\mathrm{BH}}$ and the differential cross section becomes

$$
\frac{\mathrm{d} \sigma}{\mathrm{d} \Omega} \sim \frac{E^{2} M_{\mathrm{BH}}^{4}}{M_{\mathrm{Pl}}^{8}} \sim|f(\theta)|^{2} .
$$

The scattering amplitude can be expanded in partial waves

$$
f(\theta)=\frac{1}{E} \sum_{l=0}^{\infty}\left(l+\frac{1}{2}\right) M_{l} P_{l}(\cos \theta)
$$

and unitarity demands $\left|M_{l}\right| \leqslant 1$ for any $l$. For $l=0$, we have

$$
f(\theta)=\frac{M_{0}}{2 E}
$$

and the unitarity bound reads

$$
\frac{4 E^{4} M_{\mathrm{BH}}^{4}}{M_{\mathrm{Pl}}^{8}} \sim\left|M_{0}\right|^{2} \leqslant 1 .
$$

This means that for a process with a characteristic energy scale $E \sim 1 \mathrm{GeV}$, unitarity is lost for black holes heavier than $M_{\max } \sim M_{\mathrm{Pl}}^{2} / E \sim 10^{15} \mathrm{~g}$.

So, if we want to prevent any possible participation of virtual black holes in low-energy physics, we need a more convincing explanation, since mechanisms like a new symmetry are unable to do the job. Generally speaking, one could expect that a minimum length scale acts as a regulator of the theory and somehow is capable of suppressing all the dangerous contributions above the Planck scale [21]. The realization of such a mechanism is yet to be investigated, and, unfortunately, it is unlikely that we are able to give a final answer; we would need a theory capable of providing clear predictions at the Planck scale, while at present we can at best make vague conjectures. A quite peculiar mechanism that drastically reduces any $\mathrm{B}$ and $\mathrm{L}$ violating process in the case of theories with a low gravity scale at the level to be consistent with experimental bounds has been proposed in [14] and can work even here. There the conjecture is that black holes lighter than the (effective) Planck mass must have zero charge and zero angular momentum in quantum gravity as well as classically so that proton decay is drastically suppressed. It is conjectured that black holes cannot be produced out of vacuum in violation of energy conservation, even for very short times (e.g. in $1 \mathrm{GeV}$ processes only 
$1 \mathrm{GeV}$ black holes can be produced, not very heavy ones). If we then include the idea of a minimum length, which implies a minimum black hole mass at the level of the Planck mass, black holes cannot play any role in particle physics at energies below the Planck scale. Extra dimensions might also be a solution by allowing for (otherwise unnaturally) small coupling constants [22].

\section{Conclusion}

Although a reliable theory of quantum gravity is still lacking, there are some basic ingredients (such as a minimum observable length, the generalized uncertainty principle and the idea of virtual black holes) which are commonly believed to be present in the theory of quantum gravity. It is not easy to reject the conjectures that equation (3) holds and that super-Planck mass virtual black holes predict naturally dangerous processes, clearly inconsistent with the observed universe. In particular, rapid baryon number violating processes may lead to predictions of proton decay lifetimes that are ruled out by experiment. Even if B and L violating processes are somehow forbidden, there are other problems caused by these super-Planck mass virtual black holes: heavier and heavier black holes are more and more unsuppressed, and we still have to explain how they do not alter branching ratios predicted by the standard model of particle physics. We would like to stress that such dangerous processes are expected in the constructed effective theory for virtual black holes that do not violate unitarity and hence the prediction is presumebly reliable. The problem is even more evident if we recall the peculiar predictions that can be easily deduced from the standard uncertainty principle (stability of atoms, lifetime of unstable states, stability of degenerate stars, etc): if the idea of the generalized uncertainty principle and of the spacetime foam picture are basically correct, why can we not put them together and believe in their predictions?

In this paper, we have formulated a problem which demands an answer. In particular, if we do not want to reject the generalized uncertainty principle, we have to figure out the reason for which dangerous predictions cannot occur. One possibility is to solve the puzzle with common ingredients in particle physics, say the introduction of a symmetry or something similar. The most radical case is instead that there is something wrong in what we believe to find in the theory of quantum gravity.

We believe that the study of the present problem may shed new light on the search for a theory of quantum gravity and for a unified description of gravitational and non-gravitational interactions. In addition to this, the emerging picture may be also interesting from the experimental point of view, allowing for a proton lifetime not too far from present experimental bounds. The standard description of black holes is usually expected to break down below some minimum mass $M_{\text {min }}$, which is not too much larger than $M_{\mathrm{Pl}}$. In this case, we can imagine the situation in which there is a suppression mechanism that works for dangerous heavy black holes, yet may not hold for purely quantum mechanically objects just one or two orders of magnitude heavier than the Planck mass. For example, if the contribution from black holes with a mass $M_{\mathrm{BH}} \sim 10^{2}-10^{3} M_{\mathrm{Pl}}$ is not too suppressed, the proton lifetime could be on the verge of present experimental limits.

\section{Acknowledgments}

We wish to thank Alexander Dolgov, David Garfinkle, Sabine Hossenfelder, Tsvi Piran and Dejan Stojkovic for useful comments and suggestions. CB is supported in part by NSF under grant PHY-0547794 and by DOE under contract DE-FG02-96ER41005 and thanks the MCTP 
for hospitality during his visit. KF acknowledges support from the DOE and the MCTP via the University of Michigan.

\section{References}

[1] Padmanabhan T 1987 Class. Quantum Grav. 4 L107 Amati D, Ciafaloni M and Veneziano G 1989 Phys. Lett. B 21641

Konishi K, Paffuti G and Provero P 1990 Phys. Lett. B 234276

Maggiore M 1993 Phys. Lett. B 30465 (Preprint hep-th/9301067)

Scardigli F 1999 Phys. Lett. B 45239 (Preprint hep-th/9904025)

Adler R J and Santiago D I 1999 Mod. Phys. Lett. A 141371 (Preprint gr-qc/9904026)

Bambi C and Urban F R 2008 Class. Quantum Grav. 25095006 (Preprint arXiv:0709.1965)

Bambi C 2008 Class. Quantum Grav. 25105003 (Preprint arXiv:0804.4746)

[2] Garay L J 1995 Int. J. Mod. Phys. A 10145 (Preprint gr-qc/9403008)

[3] Mead C A 1964 Phys. Rev. 135 B849

Itzhaki N 1994 Phys. Lett. B 328274 (Preprint hep-th/9404123)

[4] Nikolic H 2007 Found. Phys. 371563 (Preprint quant-ph/0609163)

[5] Dolgov A D 2004 Preprint hep-ph/0411283

[6] Hossenfelder S 2007 Phys. Rev. D 75105005 (Preprint hep-th/0702016)

[7] Bruno N R, Amelino-Camelia G and Kowalski-Glikman J 2001 Phys. Lett. B 522133 (Preprint hep-th/0107039) Magueijo J and Smolin L 2002 Phys. Rev. Lett. 88190403 (Preprint hep-th/0112090)

[8] Gibbons G W and Hawking S W 1977 Phys. Rev. D 152752

Hawking S W 1978 Nucl. Phys. B 144349

[9] Wheeler J A 1964 Relativity Groups and Topology ed B S DeWitt and C M DeWitt (New York: Gordan and Breach)

Perry M J 1977 Unification of Elementary Forces and Gauge Theories ed D B Cline and F E Mills (London: Harwood)

[10] 't Hooft G 1990 Nucl. Phys. B 335138

Susskind L 1993 Preprint hep-th/9309145

Russo J G and Susskind L 1995 Nucl. Phys. B 437611 (Preprint hep-th/9405117)

[11] Holzhey C F E and Wilczek F 1992 Nucl. Phys. B 380447 (Preprint hep-th/9202014)

Duff M J and Rahmfeld J 1995 Phys. Lett. B 345441 (Preprint hep-th/9406105)

Sen A 1995 Mod. Phys. Lett. A 102081 (Preprint hep-th/9504147)

[12] Stojkovic D, Adams F C and Starkman G D 2005 Int. J. Mod. Phys. D 142293 (Preprint gr-qc/0604072)

[13] Adams F C, Kane G L, Mbonye M and Perry M J 2001 Int. J. Mod. Phys. A 162399 (Preprint hep-ph/0009154)

[14] Bambi C, Dolgov A D and Freese K 2007 Nucl. Phys. B 76391 (Preprint hep-ph/0606321) Bambi C 2007 Preprint arXiv:0710.0235

[15] Giddings S B 1994 Phys. Rev. D 49947 (Preprint hep-th/9304027) Giddings S B 1995 Phys. Rev. D 516860 (Preprint hep-th/9412159)

[16] Bambi C 2008 Preprint arXiv:0803.2467

[17] Vilenkin A 1979 Phys. Rev. D 20373

Dolgov A D, Maeda H and Torii T 2002 Preprint hep-ph/0210267

Dolgov A and Pelliccia D N 2007 Phys. Lett. B 65097 (Preprint hep-ph/0610421)

[18] Krauss L M and Wilczek F 1989 Phys. Rev. Lett. 621221

[19] Faraggi A E 2001 Phys. Lett. B 499147 (Preprint hep-ph/0011006)

[20] Pawl A 2005 J. High Energy Phys. JHEP03(2005)034 (Preprint hep-th/0501005)

[21] Hossenfelder S 2008 Class. Quantum Grav. 25038003 (Preprint arXiv:0712.2811)

[22] Arkani-Hamed N and Schmaltz M 2000 Phys. Rev. D 61033005 (Preprint hep-ph/9903417) 